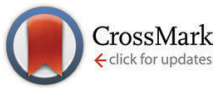

Cite this: J. Mater. Chem. C, 2016, 4, 1228

Received 28th November 2015, Accepted 4th January 2016

DOI: $10.1039 / c 5 t c 04008 f$

www.rsc.org/MaterialsC

\title{
The synthesis of novel AIE emitters with the triphenylethene-carbazole skeleton and para-I meta-substituted arylboron groups and their application in efficient non-doped OLEDs $\dagger$
}

\author{
Heping Shi, $\ddagger^{a}$ Dehua Xin, $\ddagger^{a}$ Xinggui Gu, ${ }^{b}$ Pengfei Zhang, ${ }^{b}$ Huiren Peng, ${ }^{c}$ \\ Shuming Chen, ${ }^{* c}$ Gengwei Lin, ${ }^{d}$ Zujin Zhao*d and Ben Zhong Tang ${ }^{* b d}$
}

\begin{abstract}
Four novel aggregation-induced emission (AIE)-active luminogens ( $p$-DPDECZ, $p$-DBPDECZ, $m$-DPDECZ and $m$-DBPDECZ) with triphenylethene-carbazole skeleton and para-/meta-substituted arylboron groups have been synthesized. Their structures are fully characterized using elemental analysis, mass spectrometry and proton nuclear magnetic resonance spectroscopy. The thermal stabilities, photophysical properties, electronic structures, and electrochemical properties of these molecules are investigated systematically using thermal analysis, UV-vis absorption spectroscopy, fluorescence spectroscopy, theoretical calculation and electrochemical methods. The effects of donor-acceptor interaction and conjugation degree on the photoluminescent and electroluminescent properties of these compounds are investigated. The results show that these donor-AIE-acceptor type compounds exhibit good thermal stability and electrochemical stability as well as AIE properties. Non-doped fluorescent OLEDs fabricated by using para-linked $p$-DPDECZ as an emitting layer emits a green light with a turn-on voltage of $4.8 \mathrm{~V}$, a maximum brightness of $30210 \mathrm{~cd} \mathrm{~m}^{-2}$ and a maximum current efficiency of $9.96 \mathrm{~cd} \mathrm{~A}^{-1}$. While the OLED prepared with meta-linked $m$-DBPDECZ exhibits efficient blue light emission with a maximum current efficiency of $4.49 \mathrm{~cd} \mathrm{~A}^{-1}$ and a maximum luminance of $16410 \mathrm{~cd} \mathrm{~m}^{-2}$. The electroluminescence properties of these compounds demonstrate their potential application in OLEDs.
\end{abstract}

\section{Introduction}

Organic light-emitting diodes (OLEDs) have drawn considerable attention owing to their potential applications in full-colour flat panel displays and solid state lighting since Tang's innovative work of efficient bilayer electroluminescent devices in 1987. ${ }^{1,2}$ The synthesis of new luminescent materials and optimization of device structures have been developed as the two ways to get

\footnotetext{
${ }^{a}$ School of Chemistry and Chemical Engineering, Shanxi University, Taiyuan 030006, P. R. China. E-mail: hepingshi@sxu.edu.cn

${ }^{b}$ Department of Chemistry, Institute for Advanced Study, Division of Biomedical Engineering, Division of Life Science, State Key Laboratory of Molecular Neuroscience, Institute of Molecular Functional Materials, The Hong Kong University of Science and Technology, Clear Water Bay, Kowloon, Hong Kong, China. E-mail: tangbenz@ust.hk

${ }^{c}$ Department of Electrical and Electronic Engineering, South University of Science and Technology of China, Shenzhen, Guangdong, 518055, P. R. China. E-mail: chen.sm@sustc.edu.cn

${ }^{d}$ State Key Laboratory of Luminescent Materials and Devices, South China University of Technology, Guangzhou, Guangdong, 510640, P. R. China. E-mail: mszjzhao@scut.edu.cn

$\dagger$ Electronic supplementary information (ESI) available. See DOI: 10.1039/c5tc04008f

\$ Heping Shi and Dehua Xin contributed equally to this work.
}

efficient and long-life devices, and the light-emitting materials play a vital role in the performance of the devices. So far, scientists have made great efforts in designing and synthesizing luminescent materials, now with a variety of high-performance light-emitting materials on hand..$^{3-8}$ These light-emitting materials can be divided into two types: fluorescent materials and phosphorescent materials. ${ }^{9-11}$ The phosphorescent OLEDs (PHOLEDs) have been demonstrated with an internal quantum efficiency of nearly $100 \%$, which is much higher than the fluorescent OLEDs. ${ }^{12-14}$ But the PHOLEDs usually suffer from high cost and rarity of the noble-metal complex. Besides, the PHOLEDs are mainly doped devices, whereas the non-doped devices are more attractive because they are easier to be employed for mass production and the devices are more reliable. ${ }^{15,16}$ Thus, it is of great value to develop efficient fluorescent materials. So far, high-performance fluorescent emitters for non-doped OLEDs are still scarce since most materials do not possess two crucial prerequisites; namely, showing high emission efficiency in the solid state while having good charge transporting properties.

Most thin films prepared from traditional fluorophores are usually non-emissive or weakly emissive due to the strong 
intermolecular $\pi-\pi$ stacking interactions and radiationless decay in the aggregated state (this phenomenon is known as aggregation caused quenching, ACQ). ${ }^{17}$ Since the emitters in the OLEDs were used as solid state, it is thus required to overcome the ACQ effect in order to manufacture non-doped electroluminescence devices. Many methods have been adopted to alleviate the ACQ effect, but only limited results were achieved. ${ }^{18-21}$ In 2001, a novel luminescent phenomenon named aggregationinduced emission (AIE) was found by Ben Zhong Tang et al., ${ }^{22}$ which offers a possibility to solve the ACQ effect. Different from other methods, the AIE effect takes advantage of the molecule aggregation rather than avoids it, which means that the luminous intensity of AIE materials was enhanced with the increase of aggregation degree. In dilute solutions, the AIE emitters are nonemissive or weakly emissive because of the non-radiative decays of the excited states which resulted from strong intermolecular rotation or vibration. In the aggregated state, the intermolecular motions were impeded, thus the AIE materials become strong solidstate light emitters. ${ }^{23}$ After Tang' work, many research groups have done a lot of work in designing novel AIE materials. ${ }^{24-26}$ Regarding their superior solid-state emission, AIE molecules are considered as perfectly suitable candidates as OLED emitting layer materials. Some AIE luminogens have shown satisfactory performance in OLEDs. $^{27-34}$

As we know, high-efficiency charge (electron and hole) transportation is a crucial requirement for OLED materials. ${ }^{35,36}$ If the emitting films possess both highly efficient charge-transporting capabilities and light-emission with good quantum yields, the construction process of the devices would be tremendously simplified and consequently lower the cost of furnishing OLEDs. ${ }^{37,38}$ One common strategy to improve the charge-transporting capability is to introduce electro-donor and electron-acceptor units to the chromophore. ${ }^{39,40}$ Qin et al. designed an AIE-active compound with excellent charge-transport properties, namely $\mathrm{N}, \mathrm{N}$-di(1-naphthyl)- $N, N$-diphenylbenzidine(tetraphenylethene-NPB). The non-doped device based on tetraphenylethene-NPB exhibited efficient charge-transporting and electroluminescence properties with a maximum current efficiency of $13.1 \mathrm{~cd} \mathrm{~A}^{-1} \cdot{ }^{41}$ Liu et al. synthesized and investigated a series of triphenylaminetetraphenylethene oligomers and polymers, the device exhibited a maximum current efficiency of $11.2 \mathrm{~cd} \mathrm{~A}^{-1} \cdot{ }^{42}$ Chen et al. designed and synthesized two new tetraphenylethene derivatives, tetraphenylethene-NB and tetraphenylethene-PNPB, which exhibited balanced charge-transporting capabilities due to the diphenylamino and dimesitylboryl units. Their non-doped device showed excellent current efficiency of $16.2 \mathrm{~cd} \mathrm{~A}^{-1}$. $^{3}$

As one of the earliest reported AIE structures, triphenylethene (TPE) has attracted much attention because of the facile synthesis and versatile functionalization. ${ }^{44,45}$ Based on TPE, many efficient electroluminescent materials have been reported in recent years. For example, Adachi et al. synthesized two TPE-based (PDA-TPE and TPA-TPE) molecules with strong AIE characteristics. They utilized these materials to fabricate efficient OLEDs, the devices exhibited a

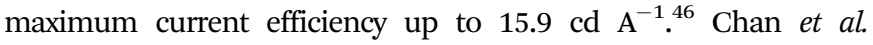
designed and synthesized two TPE-based carbazole compounds by incorporating 9-carbazolyl and 3-carbazolyl into the 4-position of TPE, the optimized devices made from the materials exhibited blue-light emission with high luminance and the current efficiency up to $11700 \mathrm{~cd} \mathrm{~m}^{-2}$ and $7.5 \mathrm{~cd} \mathrm{~A}^{-1}$, respectively. ${ }^{47} \mathrm{Chi}$ et al. synthesized a series of aggregation-induced emission enhancement molecules by combining TPE, triphenylamine and thiadiazole into one molecule, the fabricated non-doped red OLEDs showed a maximum current efficiency of $6.81 \mathrm{~cd} \mathrm{~A}^{-1}$. $^{48}$ These remarkable device performances aroused our interest. We assumed that by introducing strong electron-donor and electron-acceptor to the TPE structure, the resultant compound is likely to be endowed with good charge-transporting capabilities and high efficiency emitting properties. Carbazole and arylboron moieties have been widely used as optoelectronic materials due to the excellent electron-donating and -withdrawing properties, respectively. We design to combine the TPE, carbazole and arylboron units into one molecule to get new electroluminescent emitters and enlarge the AIE library.

In this paper, we report four novel TPE-carbazole emitters with para- and meta-substituted arylboron groups, abbreviated as $p$-DPDECZ, $p$-DBPDECZ, $m$-DPDECZ and $m$-DBPDECZ (Fig. 1 ). In these molecules, an $N$-ethyl-carbazole group was attached to the 4-position of the TPE unit, then dimesitylboron and (dimesitylboranyl)phenyl groups were linked to the para- or meta-position of the 3-phenyl of TPE, respectively. We investigated the effects of donor-acceptor interaction and conjugation degree on the photoluminescent and electroluminescent properties of these compounds. Our research results proved that construction of donor-AIE-accepter type compounds is a method for obtaining efficient emitters. In addition, by connecting the electron-donor units with electron-acceptor moieties with different linkage modes, we were able to control the degree of twisting of the molecules, thereby getting materials with different colors. The non-doped

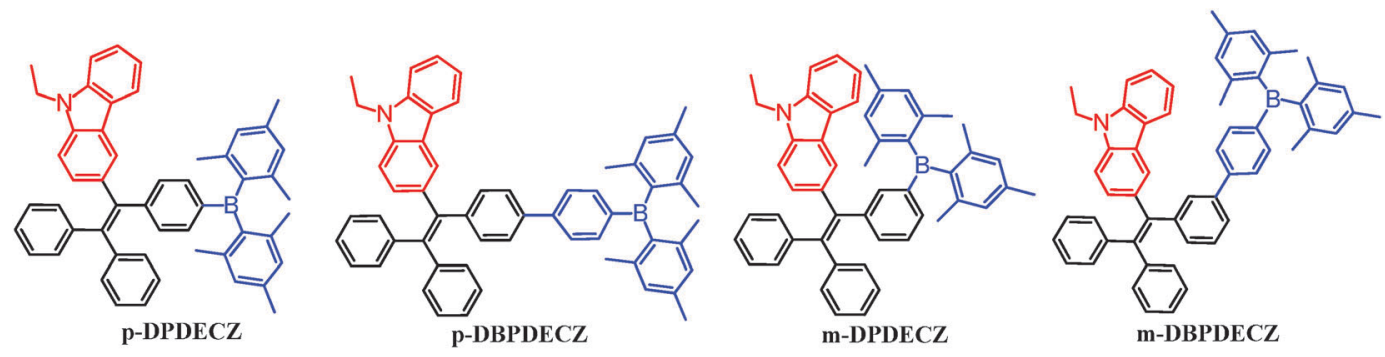

Fig. 1 Chemical structures of $p$-DPDECZ, $p$-DBPDECZ, $m$-DPDECZ and $m$-DBPDECZ. 
OLED devices prepared with these materials also showed good performance.

\section{Results and discussion}

$p$-DPDECZ and $p$-DBPDECZ were synthesized according to the synthetic routes shown in Scheme 1 . First, compound 1 was synthesized by the Friedel-Crafts reaction of $\mathrm{N}$-ethylcarbazole with 4-bromobenzoylchloride in dichloromethane. Followed by a nucleophilic addition of lithiated diphenylmethane and a subsequent acid-catalyzed elimination of water, compound 2 was prepared in good yield. Finally, $p$-DPDECZ was obtained by reacting lithiated compound 2 with dimesitylboron fluoride at $-78{ }^{\circ} \mathrm{C}$ in $69.2 \%$ yield. $p$-DBPDECZ was synthesized via the Suzuki coupling reaction of compound 2 with [4-(dimesitylboranyl)phenyl]boronic acid, catalyzed by a palladium complex in THF/ water mixtures. The synthesis routes of $m$-DPDECZ and $m$-DBPDECZ are similar to that of $p$-DPDECZ and $p$-DBPDECZ, the only difference is that 4-bromobenzoylchloride is replaced with 3-bromobenzoylchloride. Detailed synthetic procedures and characterization data are described in the ESI. $\dagger$ All the compounds are readily soluble in common organic solvents, such as tetrahydrofuran (THF), toluene, chloroform and dichloromethane, but insoluble in water.

Since good thermal properties of the light-emitting materials are beneficial to the stability and lifetime of OLED devices, we evaluated the thermal properties of these compounds by thermogravimetric analysis (TGA) and differential scanning calorimetry (DSC) measurements. The TGA and DSC curves of these AIE luminogens are shown in Fig. S1 (ESI $\dagger$ ). The decomposition temperature $\left(T_{\mathrm{d}}\right)$ of $p$-DPDECZ is $301{ }^{\circ} \mathrm{C}$ accounting for a $5 \%$ loss of the initial weight. The $T_{\mathrm{d}}$ of $p$-DBPDECZ is $274{ }^{\circ} \mathrm{C}$. The meta-linked compounds $m$-DPDECZ and $m$-DBPDECZ possess high $T_{\mathrm{d}}$ as 328 and $365{ }^{\circ} \mathrm{C}$. These twisted molecules also possess good morphological stability, as confirmed by their high glasstransition temperature $\left(T_{\mathrm{g}}\right)$ of $100,115,138,146{ }^{\circ} \mathrm{C}$, respectively, which should arise from the rigid aromatic scaffolds of the TPE units. The data are summarized in Table 1 . The high $T_{\mathrm{d}}$ and $T_{\mathrm{g}}$ values show that these new compounds have good thermal and morphological stability, thus fulfilling the thermo-physical demands for electroluminescent materials.

The optical properties of these AIE luminogens were investigated by UV-vis spectra and photoluminescence spectra. Fig. S2 (ESI $\dagger$ ) shows the UV-vis absorption spectra of these AIE luminogens in THF solvents $(10 \mu \mathrm{M})$. As illustrated in Fig. S2 (ESI $\dagger)$, the spectra of the four compounds exhibit different features. $p$-DPDECZ and $m$-DPDECZ exhibit three similar absorption bands. The strong absorption peaks in the wavelength of $300 \mathrm{~nm}$ originate from the $\pi-\pi^{*}$ transition of the TPE moiety, the two weak absorption bands at about $330 \mathrm{~nm}$ and $360 \mathrm{~nm}$ are attributed to the $\pi-\pi^{*}$ transition of the carbazole scaffold and the intramolecular charge transfer (ICT) from carbazole to dimesitylboron, respectively. $p$-DBPDECZ and $m$-DBPDECZ exhibit a similar absorption peak, the peak located at $300 \mathrm{~nm}$ corresponds to the $\pi-\pi^{*}$ transition of the TPE moiety. But the ICT absorption of $p$-DBPDECZ and $m$-DBPDECZ were blue-shifted and has partially merged with the absorption band of the $\pi-\pi^{*}$ transition of the backbone, indicating the weaker donor-acceptor interaction than that of $p$-DPDECZ and $m$-DPDECZ, respectively. ${ }^{49}$
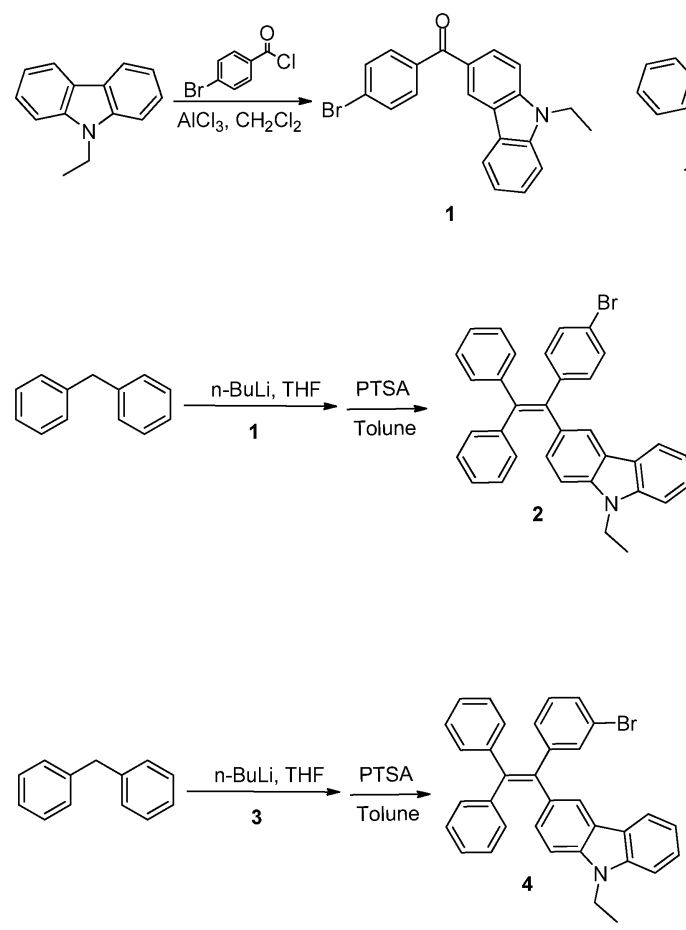
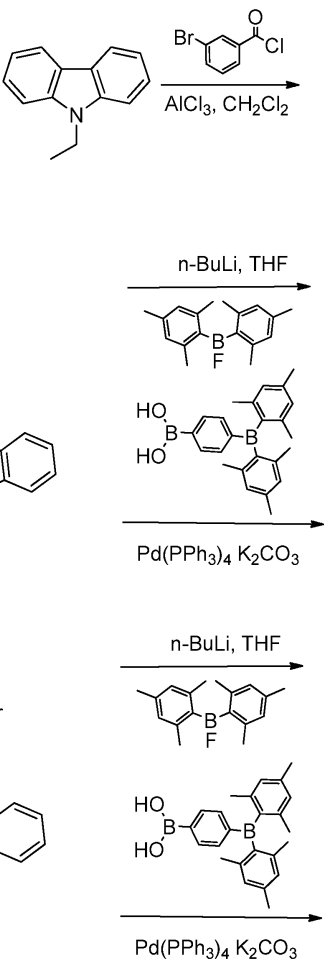

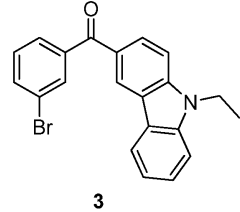

p-DPDECZ

p-DBPDECZ

m-DPDECZ

m-DBPDECZ

Scheme 1 Synthetic routes of the AIE luminogens. 
Table 1 Physical properties of the four compounds

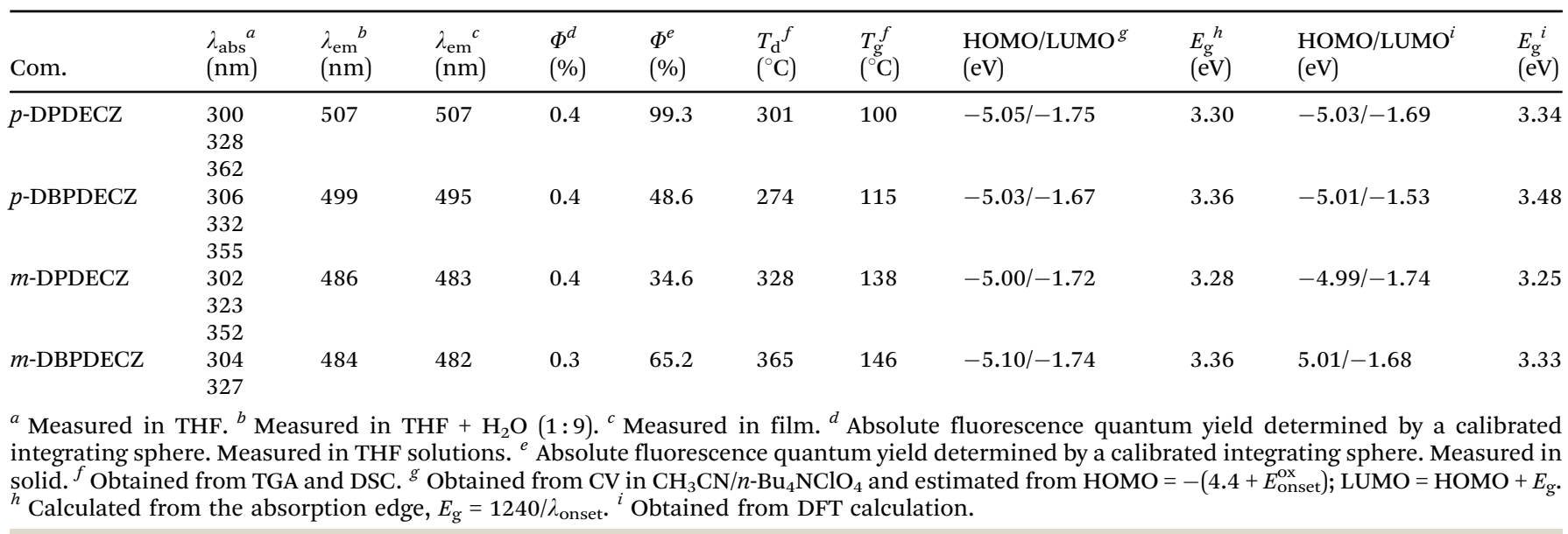

The ICT absorption peak of $m$-DPDECZ is slightly blue-shifted than that of $p$-DPDECZ, which may result from the stronger ICT effect of the para-linked molecule than the meta-linked molecule. The difference of the absorption spectra between $p$-DBPDECZ and $m$-DBPDECZ testified this point. It can be observed that $p$-DBPDECZ exhibits ICT absorption while $m$-DBPDECZ has no clear ICT absorption or the ICT absorption was blue-shifted as merged with the absorption of $\pi-\pi^{*}$ transition of the carbazole scaffold. At last, the absorption spectra of the four compounds in various solvents were measured. As shown in Fig. S3 (ESI $\dagger$ ), all the compounds show similar absorption in various solvents, indicating their electronic structures in the ground state in various solvents are independent of the polarity of solvents. The data of absorption spectra are summarized in Table 1.

In order to investigate the photoluminescence behaviors of these four compounds, their fluorescence spectra were measured in THF-water mixtures with different water volume fractions $\left(f_{\mathrm{w}}\right)$. Herein, THF acts as the solvent and water acts as the non-solvent. As shown in Fig. 2(a)-(d), the spectra of $p$-DPDECZ, $p$-DBPDECZ and $m$-DBPDECZ were similar, while $m$-DPDECZ exhibited some differences. $p$-DPDECZ, $p$-DBPDECZ and $m$-DBPDECZ were practically non-emissive in pure THF solution for the loss of energy by intramolecular rotations in the excited state. The fluorescence quantum yields $(\Phi)$ for $p$-DPDECZ, $p$-DBPDECZ, $m$-DPDECZ and $m$-DBPDECZ in dilute THF solutions are as low as $0.4,0.4$ and 0.4 and $0.3 \%$, respectively, measured by an integrating sphere. The emission spectra remained almost unchanged when the water volume fraction was gradually increased from $0 \%$ to $60 \%$. Further incremental increase of the water content led to an increase of the emission intensity due to the formation of nanoparticles reducing the freedom of intramolecular rotation. The spectra of $m$-DPDECZ in high water content solutions $\left(f_{\mathrm{w}}>80 \%\right)$ were similar to that of $p$-DPDECZ, $p$-DBPDECZ and $m$-DBPDECZ. However, $m$-DPDECZ showed distinctive features in low water content solutions. In pure THF solution, $m$-DPDECZ exhibits a tiny emission peak centered at $393 \mathrm{~nm}$, which is assigned to the twisted intramolecular charge transfer (TICT) emission. As the water content increases, the polarity of the solvents increases. With the water volume fraction gradually increasing from $0 \%$ to
$70 \%$, the intensity of the TICT peak was enhanced and the maximum peak was shifted from $393 \mathrm{~nm}$ to $405 \mathrm{~nm}$. When the water content is further increased, the TICT emission vanished on formation of aggregates. We inferred that the unique TICT peak of $m$-DPDECZ may be due to its more twisted structure and steric crowdedness than $p$-DPDECZ, $p$-DBPDECZ and $m$-DBPDECZ. The maximum emission peaks of $p$-DPDECZ, $p$-DBPDECZ, $m$-DPDECZ and $m$-DBPDECZ in 95\% (water/THF) solution were located at 507, 499, 486 and $484 \mathrm{~nm}$, respectively (Table 1). It is suggested that the phenyl of the (dimesitylboranyl)phenyl interrupts the conjugation of the molecular structure, thus the ICT effect is weaker in $p$-DBPDECZ. Thus the emission of $p$-DBPDECZ was $8 \mathrm{~nm}$ blue-shifted than $p$-DPDECZ. While the meta-linked $m$-DPDECZ and $m$-DBPDECZ both have a very weak ICT effect, the emission bands are at almost the same region. meta-Substituted $m$-DPDECZ showed blue-shifted emission than para-substituted $p$-DPDECZ because that the meta-linked compound is more twisted than the para-linked compounds, thus it has smaller conjugation degree. ${ }^{31}$ Fig. 2(e) depicts the plots of relative photoluminescence $\left(\left(I / I_{\mathrm{o}}\right)-1\right)$ against the water volume fractions (in the maximum emission peak). From the solubilized state in THF to the aggregate suspension $\left(f_{\mathrm{w}}=95 \%\right)$, the fluorescence intensity of the four compounds increased by an approximate factor of 191, 139, 91 and 251. Thus, we could unambiguously demonstrate the AIE-behaviors of these donorAIE-acceptor compounds. The fluorescence spectra of these solid compounds are obtained by measuring their films and depicted in Fig. $\mathrm{S} 4$ (ESI $\dagger$ ). As shown there, $p$-DPDECZ emits green light with a maximum peak $\left(\lambda_{\max }\right)$ at $507 \mathrm{~nm}$, which is consistent with the emission peaks recorded from the THF-water mixtures. The maximum emission peaks of $p$-DBPDECZ, $m$-DPDECZ, and $m$-DBPDECZ were located at 495, 483 and $482 \mathrm{~nm}$, respectively (Table 1). The solid state emission spectra of these compounds were in good agreement with their fluorescence spectra in solutions. The $\Phi$ values of $p$-DPDECZ, $p$-DBPDECZ, $m$-DPDECZ and $m$-DBPDECZ in the solid state are 99.3, 48.6, 34.6 and $65.2 \%$, respectively, which demonstrated that these AIE luminogens are high efficiency solid-state emitters. The PL properties of these compounds indicate their suitability for the fabrication of OLEDs. 

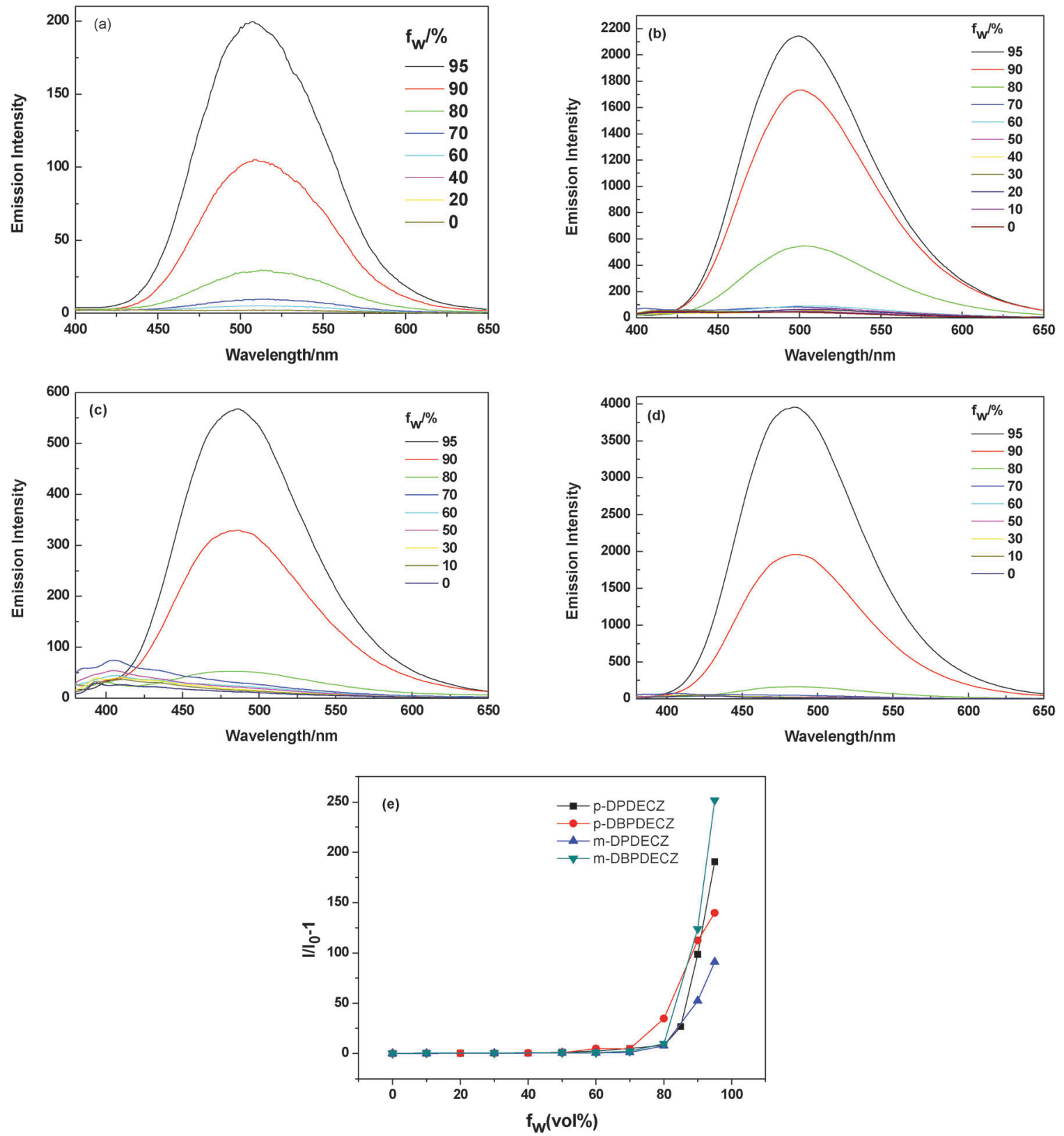

Fig. 2 PL spectra of $p$-DPDECZ (a), p-DBPDECZ (b), $m$-DPDECZ (c) and $m$-DBPDECZ (d) in various water contents of THF-water mixtures at excitation of $350 \mathrm{~nm}$. (e) Plot of $\left(I / I_{0}\right)-1$ against water volume fraction $\left(f_{\mathrm{w}}\right)$, where $I_{0}$ and $/$ are the PL intensities without and with water in the THF-water mixtures. The intensity was monitored at excitation/emission wavelengths of $350 \mathrm{~nm} / \lambda_{\max }$.

The redox properties and the molecular orbital energies of these compounds were investigated by cyclic voltammetry (CV). Fig. S5 (ESI $\dagger$ ) shows the cyclic voltammogram (CV) of these compounds. $p$-DPDECZ exhibits two reversible oxidation and reduction peaks. The oxidation peaks at $0.97 \mathrm{~V}$ and $1.23 \mathrm{~V}$ probably originated from the oxidation of the TPE and carbazole groups. Reduction peaks at $-0.63 \mathrm{~V}$ and $-1.65 \mathrm{~V}$ were assigned to the reduction of the TPE and dimesitylboron moieties, respectively. Similar CV curves were observed for $p$-DBPDECZ, $m$-DPDECZ and $m$-DBPDECZ. The onset oxidation potentials of $p$-DPDECZ, $p$-DBPDECZ, $m$-DPDECZ and $m$-DBPDECZ are observed to be $0.65,0.63,0.60$ and $0.70 \mathrm{~V}$, respectively. Thus the
HOMO energy levels can be calculated with the empirical equation: $\mathrm{HOMO}=-\left(E_{\mathrm{ox}}+4.40\right) \mathrm{eV}$, the HOMO energy levels are $-5.05,-5.03$, -5.00 and $-5.10 \mathrm{eV}$, respectively. The LUMO energy levels of $p$-DPDECZ, $p$-DBPDECZ, $m$-DPDECZ and $m$-DBPDECZ are calculated according to the optical band gap energy (determined from the absorption onset wavelength) and $E_{\mathrm{HOMO}}$, the calculated LUMO energy are $-1.75,-1.67,-1.72$ and $-1.74 \mathrm{eV}$, respectively. All the data are also summarized in Table 1 . The CV curves remain unchanged under multiple successive potential scans, indicating the excellent redox stability of these compounds.

To get further insight into the photophysical properties of these compounds, the optimized molecular geometry of these 
HOMO
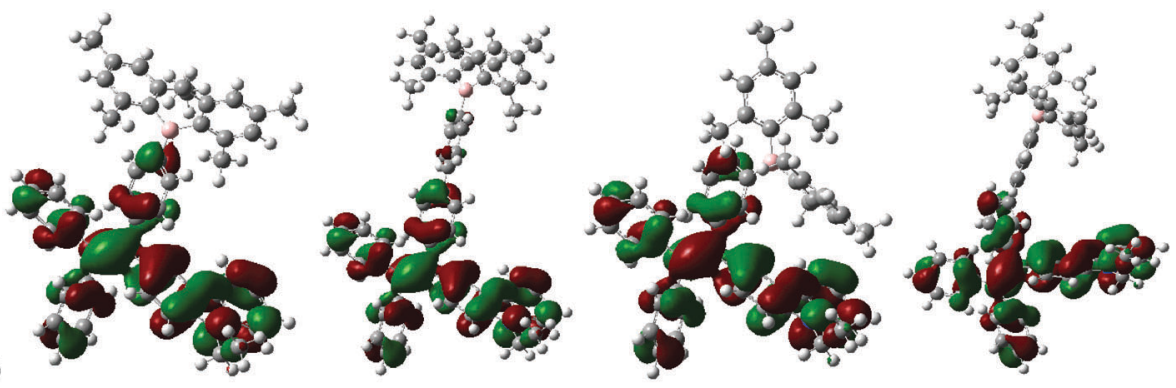

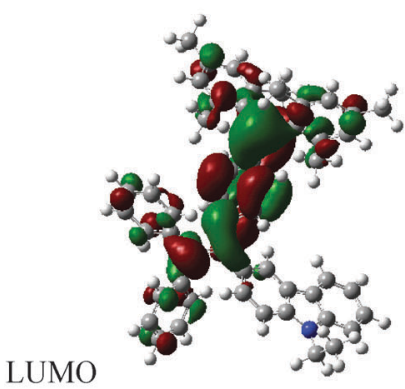

(a)

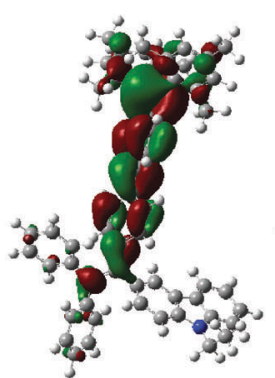

(b)

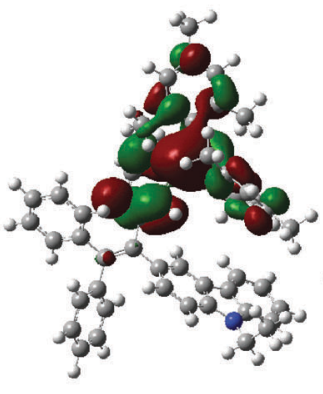

(c)

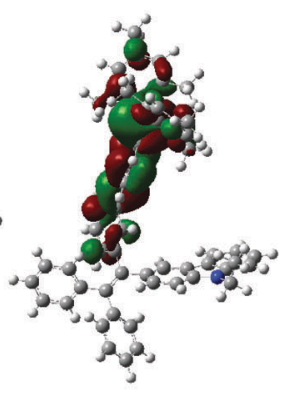

(d)

Fig. 3 The optimal structure and HOMO and LUMO distributions of $p$-DPDECZ (a), $p$-DBPDECZ (b), $m$-DPDECZ (c) and $m$-DBPDECZ (d).

compounds have been determined using density functional theory (DFT) calculations at the B3LYP/6-31G(d,p) level with the Gaussian 03 program (ESI $\dagger$ ). The orbital distributions of the highest occupied molecular orbital (HOMO) and the lowest unoccupied molecular orbital (LUMO) are shown in Fig. 3. As illustrated in Fig. 3, the structure of $p$-DPDECZ adopts propellershaped conformations with rotational freedom, which allows for the facile conversion of the energy in the excited state via a non-radiative pathway. Meanwhile, the non-planar conformation of $p$-DPDECZ should prevent close molecular packing - especially stacking interactions - in the solid state, leading to efficient fluorescence in the condensed solid states. $m$-DPDECZ adopts a more twisted conformation due to the meta-linkage mode. As shown in Fig. 4, the dihedral angles of the biphenyl linkages of $p$-DBPDECZ and $m$-DBPDECZ are $34.74^{\circ}$ and $35.86^{\circ}$, respectively. $p$-DBPDECZ and $m$-DBPDECZ adopt more twisted conformations than $p$-DPDECZ and $m$-DPDECZ because of the dihedral angles in the biphenyl linkages. The electron distribution of the HOMO of $p$-DPDECZ is located mainly on the carbazole ring and partly on the phenyl units of TPE, while the LUMO is mostly located on the dimesitylboron moiety and to a lesser extent on the TPE scaffold. In contrast to $p$-DPDECZ, the spatial distributions of the HOMO and the LUMO is less overlapped in $p$-DBPDECZ, which is attributed to its more twisted conformation and ineffective conjugation. ${ }^{43}$ The HOMO and LUMO orbitals of $m$-DPDECZ and $m$-DBPDECZ are even more detached due to the more twisted conformations and ineffective conjugations. The separation of the HOMO and LUMO of $p$-DBPDECZ, $m$-DPDECZ and $m$-DBPDECZ demonstrated that their donor-acceptor interaction are weaker than that in $p$-DPDECZ. ${ }^{43}$ And it is beneficial to charge balance and exciton recombination in devices. ${ }^{50}$ This sort of HOMO-LUMO distribution is typical for intramolecular charge transfer. The calculated
HOMO energy levels of $p$-DPDECZ, $p$-DBPDECZ, $m$-DPDECZ and $m$-DBPDECZ are $-5.03,-5.01,-4.99$ and $-5.01 \mathrm{eV}$, respectively. The calculated LUMO energy levels are $-1.69,-1.53,-1.74$ and $-1.68 \mathrm{eV}$, respectively. The energy gaps obtained from the calculated HOMO and LUMO energy levels are 3.34, 3.48, 3.25 and $3.33 \mathrm{eV}$, respectively (Table 1).

The above studies have shown these compounds possess good thermal and electrochemical stabilities as well as efficient solid-state emission. To investigate their electroluminescence properties, these compounds were employed as a light emitter layer in non-doped OLEDs. Therefore, we constructed four devices ( $\mathrm{M}$ for $p$-DPDECZ, $\mathrm{N}$ for $p$-DBPDECZ, $\mathrm{P}$ for $m$-DPDECZ, and $\mathrm{Q}$ for $m$-DBPDECZ) with the configuration of ITO/ $\operatorname{HATCN}(20 \mathrm{~nm}) / \operatorname{NPB}(40 \mathrm{~nm}) / \operatorname{EMITTER}(20 \mathrm{~nm}) / \operatorname{TPBi}(40 \mathrm{~nm}) /$ $\operatorname{LiF}(1 \mathrm{~nm}) / \mathrm{Al}(80 \mathrm{~nm})$, where dipyrazino[2,3-f: $\left.2^{\prime}, 3^{\prime}-h\right]$ quinoxaline-2,3,6,7,10,11-hexacarbonitrile (HATCN) serves as a holeinjection layer, $N, N$-bis(1-naphthyl)- $N, N$-diphenylbenzidine (NPB) and 1,3,5-tris( $N$-phenylbenzimidazol-2-yl)benzene (TPBi) work as hole-transport and electron-transport layers. The electroluminescent spectra of the four devices are shown in Fig. 5(a). Devices M, N, P and Q give electroluminescence at 524, 513, 489 and $500 \mathrm{~nm}$, respectively, which are slightly shifted compared to the emission peaks of the films and the nanoparticles in $95 \%$ aqueous mixture, indicating that the EL emission should be from the amorphous emitting layers. Device $\mathrm{M}$ and $\mathrm{N}$ show green electroluminescence with Commission International de L'Eclairage (CIE) coordinates of $(0.25,0.52)$ and $(0.23,0.46)$, respectively. The CIE coordinates are shown in Fig. 5(b). Device P and Q exhibit skyblue electroluminescence with CIE coordinates of $(0.19,0.30)$ and $(0.20,0.34)$, respectively. The current efficiency-current density curves are shown in Fig. 6. Current density-voltageluminance curves are depicted in Fig. 7. The relevant performance data are summarized in Table 2. Device M displays 


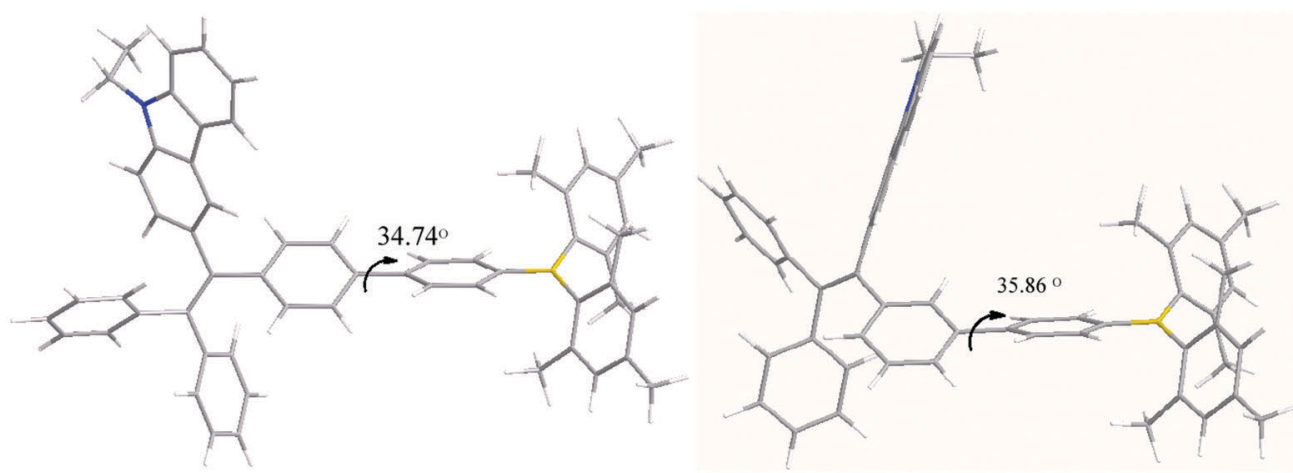

Fig. 4 The optimized structures of $p$-DBPDECZ and $m$-DBPDECZ (stick model).
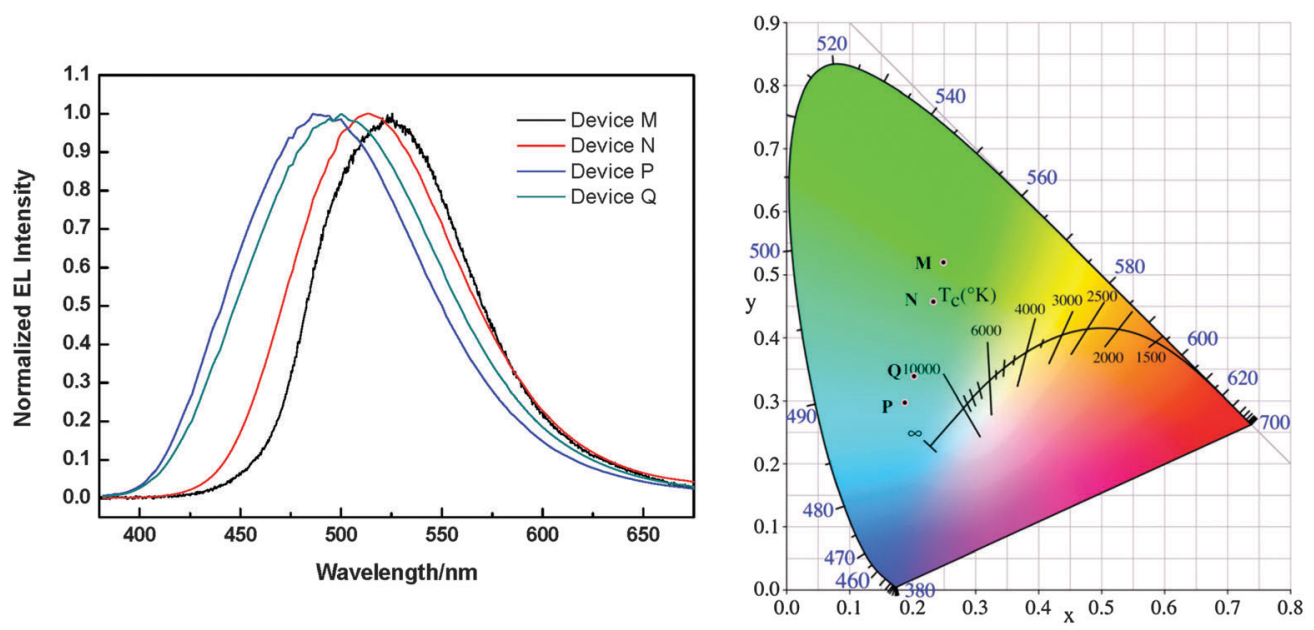

Fig. 5 (a) Electroluminescent spectra of the four devices and (b) CIE chromaticity coordinates of the four devices.

promising green electroluminescence properties with a turnon voltage of $4.80 \mathrm{~V}$, a maximum brightness of $30210 \mathrm{~cd} \mathrm{~m}^{-2}$ (at $15 \mathrm{~V}$ ) and a maximum current efficiency of $9.96 \mathrm{~cd} \mathrm{~A}^{-1}$ (at $9.2 \mathrm{~V}$ ). Device $\mathrm{N}$ shows a lower turn-on voltage of $4.2 \mathrm{~V}$, a higher maximum luminance of $65150 \mathrm{~cd} \mathrm{~m}^{-2}$ (at $15 \mathrm{~V}$ ), and a slightly lower maximum current efficiency of $8.60 \mathrm{~cd} \mathrm{~A}^{-1}$ (at $6 \mathrm{~V}$ ). The maximum external quantum efficiencies of device

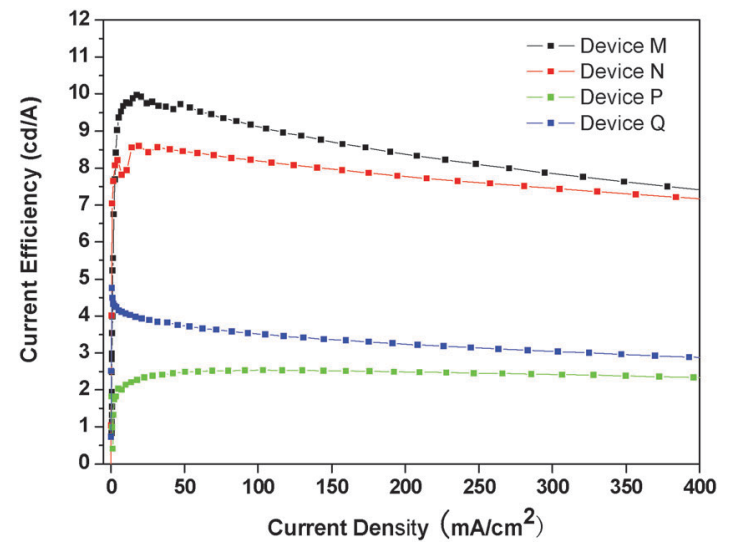

Fig. 6 Current efficiency-current density curves of the four devices.
$\mathrm{M}$ and $\mathrm{N}$ are 2.73 and $3.28 \%$, respectively. To the best of our knowledge, such high electroluminescence performance is among the best for non-doped green fluorescent devices reported previously. ${ }^{41-43,46}$ Device $\mathrm{P}$ and $\mathrm{Q}$ exhibit much blue-shifted emissions than $\mathrm{M}$ and $\mathrm{N}$ due to the more twisted and inefficient conjugation structure of the meta-linked compounds. Device P was turned on at a voltage of $4.90 \mathrm{~V}$ and reached a luminance level of $500 \mathrm{~cd} \mathrm{~m}^{-2}$ at $7.20 \mathrm{~V}$ and a maximum luminance of $14980 \mathrm{~cd} \mathrm{~m}^{-2}$ at about $15 \mathrm{~V}$, the maximum current efficiency is $2.53 \mathrm{~cd} \mathrm{~A}^{-1}$. Device $\mathrm{Q}$ exhibits high efficiency with a turn-on voltage of $6 \mathrm{~V}$, a maximum current efficiency of $4.49 \mathrm{~cd} \mathrm{~A}^{-1}$ and a maximum luminance of $16410 \mathrm{~cd} \mathrm{~m}^{-2}$. The maximum external quantum efficiencies of devices $\mathrm{P}$ and $\mathrm{Q}$ are 1.26 and $2.16 \%$, respectively. The electroluminescent performances of devices $\mathrm{P}$ and $\mathrm{Q}$ are also very impressive. ${ }^{31}$ The strong electroluminescence of these materials may result from the efficient charge-transport capabilities of the electron-donor carbazole unit and electro-acceptor dimesitylboron moiety. It should be pointed out that the electroluminescent performances were obtained in a non-optimized test device under ordinary laboratory conditions. The device performances may be further improved by optimizing the process conditions. 

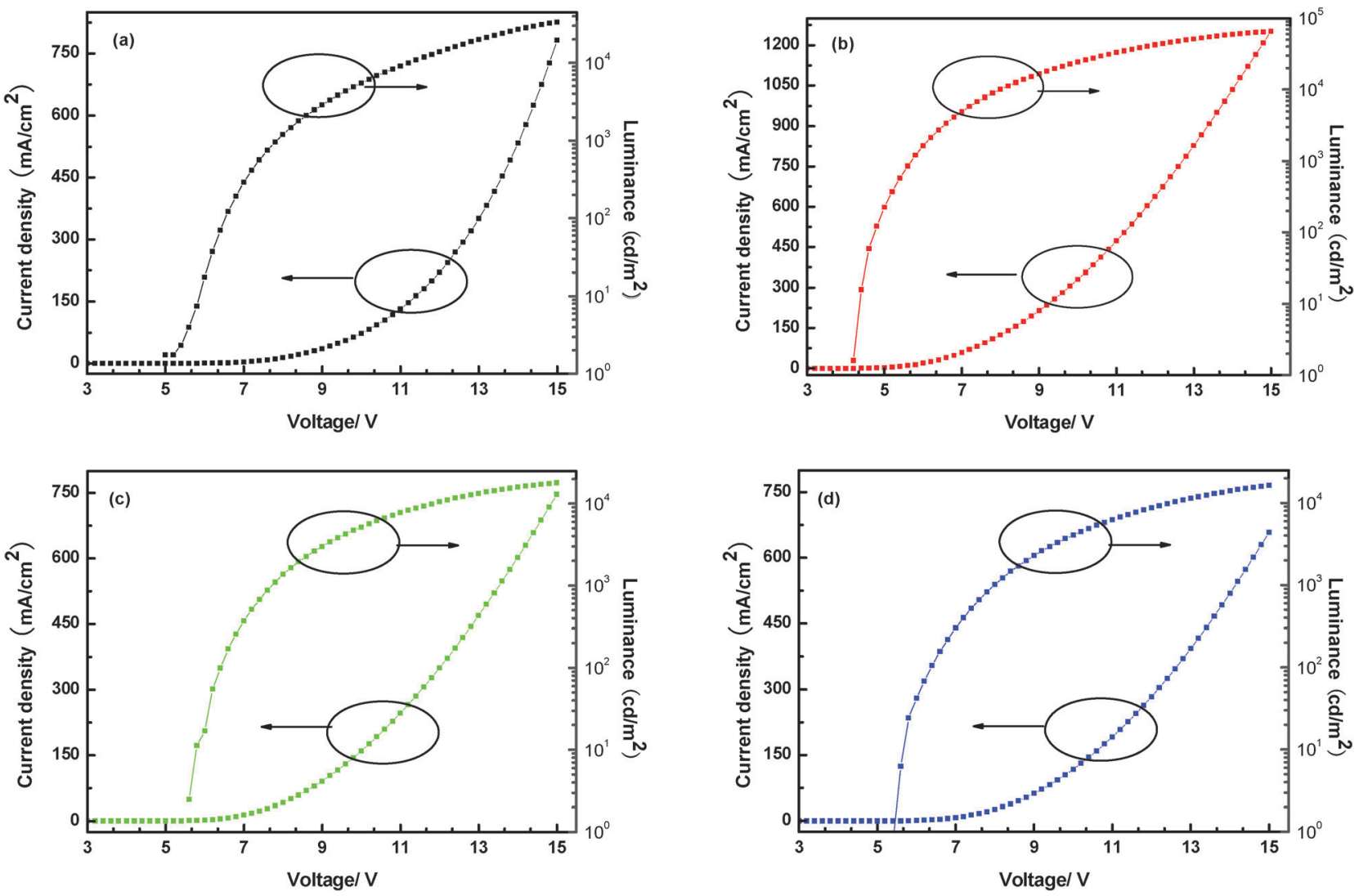

Fig. 7 Current density-voltage-luminance curves of device $M(a)$, device $N(b)$, device $P(c)$ and device $Q$ (d).

Table 2 Performances of the four devices

\begin{tabular}{|c|c|c|c|c|c|c|c|}
\hline Device & $V_{\mathrm{on}}{ }^{a}(\mathrm{~V})$ & $L_{\max }^{b}\left(\mathrm{~cd} \mathrm{~m}^{-2}\right)$ & $\mathrm{CE}_{\max }^{c}\left(\mathrm{~cd} \mathrm{~A}^{-1}\right)$ & $\mathrm{EQE}_{\max }^{d}(\%)$ & $\mathrm{PE}_{\max }^{e}\left(\operatorname{lm~} \mathrm{W}^{-1}\right)$ & $\lambda_{\mathrm{EL}}^{f}(\mathrm{~nm})$ & $\operatorname{CIE}(x, y)$ \\
\hline $\mathrm{N}$ & 4.2 & 65150 & 8.60 & 3.28 & 5.07 & 513 & $(0.23,0.46)$ \\
\hline
\end{tabular}

${ }^{a}$ Turn on voltage at a brightness of $1 \mathrm{~cd} \mathrm{~m}^{-2} \cdot{ }^{b}$ Maximum luminance. ${ }^{c}$ Maximum current efficiency. ${ }^{d}$ Maximum external quantum efficiency. ${ }^{e}$ Maximum power efficiency. ${ }^{f}$ Electroluminescence peak.

\section{Conclusion}

In summary, we synthesized four novel AIE luminogens with electron donor-acceptor structure featuring triphenylethene, carbazole and dimesitylboron scaffolds. The structure was fully characterized by ${ }^{1} \mathrm{H}$ NMR, ${ }^{13} \mathrm{C}$ NMR, MS and elemental analysis. The properties of these AIE luminogens with regard to their suitability for non-doped OLED devices were studied in a multidimensional approach involving thermogravimetric analysis (TGA), differential scanning calorimetry (DSC), electrochemical analysis, UV-vis and photoluminescence spectra analysis and quantum chemical calculations. The effects of donor-acceptor interaction and conjugation degree on the photoluminescent and electroluminescent properties are also investigated by the spectra and calculation. The results show that these AIE luminogens exhibit excellent thermal and electrochemical stabilities. And by controlling the linkage mode and the conjugation length, the emission properties of compounds can be tuned. At last, four non-doped OLED devices are prepared using these AIE luminogens as a light-emitting layer. The device fabricated with a para-linked compound exhibits a green emission with a maximum current efficiency of $9.96 \mathrm{~cd} \mathrm{~A}^{-1}$, while the device based on a meta-linked compound showed sky-blue emission with a maximum current efficiency of $4.49 \mathrm{~cd} \mathrm{~A}^{-1}$. The present results virtually demonstrate that combining an AIE-unit with charge-transporting moieties in one molecule is a versatile approach to obtain a high performance light-emitting material. Our strategy provides a new way for the development of new light-emitting materials.

\section{Acknowledgements}

This work was supported by Beijing National Laboratory for Molecular Sciences (No. BNLMS 2013031); Open Fund of the 
State Key Laboratory of Luminescent Materials and Devices, South China University of Technology (No. 2014-skllmd-09); Scientific and Technological Innovation Programs of Higher Education Institutions in Shanxi Province (No. 2014109 and 2012005); National Natural Science Foundation of China (No. 61405089); The Innovation of Science and Technology Committee of Shenzhen (No. JCYJ20140417105742713); State Key Laboratory of Structural Chemistry, Fujian Institute of Research on the Structure of Matter, Chinese Academy of Sciences (No. 20140016) and Fund of Key Laboratory of Optoelectronic Materials Chemistry and Physics, Chinese Academy of Sciences (No. 2008DP173016).

\section{References}

1 C. W. Tang and S. A. VanSlyke, Appl. Phys. Lett., 1987, 51, 913-915.

2 J. H. Burroughes, D. D. C. Bradley, A. R. Brown, R. N. Marks, K. Mackay, R. H. Friend, P. L. Burns and A. B. Holmes, Nature, 1990, 347, 539-541.

3 B. K. Shah, D. C. Neckers, J. M. Shi, E. W. Forsythe and D. Morton, Chem. Mater., 2006, 18, 603-608.

4 Q. Fang, B. Xu, B. Jiang, H. T. Fu, X. Y. Chen and A. M. Cao, Chem. Commun., 2005, 1468-1470.

5 S. Ahn, Y.-B. Cha, M. Kim, K.-H. Ahn and Y. C. Kim, Synth. Met., 2015, 199, 8-13.

6 T. W. Kwon, M. M. Alam and S. A. Jenekhe, Chem. Mater., 2004, 16, 4657-4666.

7 Z. J. Zhao, S. M. Chen, C. M. Deng, J. W. Y. Lam, C. Y. K. Chan, P. Lu, Z. M. Wang, B. B. Hu, X. P. Chen, P. Lu, H. S. Kwok, Y. G. Ma, H. Y. Qiu and B. Z. Tang, J. Mater. Chem., 2011, 21, 10949-10956.

8 Y. T. Tao, E. Balasubramaniam, A. Danel, B. Jarosz and P. Tomasik, Chem. Mater., 2001, 13, 1207-1212.

9 M. A. Baldo, D. F. O'Brien, Y. You, A. Shoustikov, S. Sibley, M. E. Thompson and S. R. Forrest, Nature, 1998, 395, 151-154.

10 M. A. Baldo, S. Lamansky, P. E. Burrows, M. E. Thompson and S. R. Forrest, Appl. Phys. Lett., 1999, 75, 4-6.

11 H. Uoyama, K. Goushi, K. Shizu, H. Nomura and C. Adachi, Nature, 2012, 492, 234-238.

12 C. Adachi, M. A. Baldo, M. E. Thompson and S. R. Forrest, J. Appl. Phys., 2001, 90, 5048-5051.

13 L. X. Xiao, S. J. Su, Y. Y. Agata, H. L. Lan and J. J. Kido, Adv. Mater., 2009, 21, 1271-1274.

14 D. B. Xia, B. Wang, B. Chen, S. M. Wang, B. H. Zhang, J. Q. Ding, L. X. Wang, X. B. Jing and F. S. Wang, Angew. Chem., Int. Ed., 2014, 53, 1048-1052.

15 T.-H. Huang, J. T. Lin, L.-Y. Chen, Y.-T. Lin and C.-C. Wu, Adv. Mater., 2006, 18, 602-606.

16 F. Wei, T. Zhang, X. C. Liu, X. Y. Li, N. Jiang, Z. W. Liu, Z. Q. Bian, Y. L. Zhao, Z. H. Lu and C. H. Huang, Org. Electron., 2014, 15, 3292-3297.

17 S. W. Thomas III, G. D. Joly and T. M. Swager, Chem. Rev., 2007, 107, 1339-1386.

18 C.-L. Chiang, S.-M. Tseng, C.-T. Chen, C.-P. Hsu and C.-F. Shu, Adv. Funct. Mater., 2008, 18, 248-257.
19 J. Wang, Y. Zhao, C. Dou, H. Sun, P. Xu, K. Ye, J. Zhang, S. Jiang, F. Li and Y. Wang, J. Phys. Chem. B, 2007, 111, 5082-5089.

20 A. C. Grimsdale, K. L. Chan, R. E. Martin, P. G. Jokisz and A. B. Holmes, Chem. Rev., 2009, 109, 897-1091.

21 J. Z. Liu, J. W. Y. Lam and B. Z. Tang, Chem. Rev., 2009, 109, 5799-5867.

22 J. D. Luo, Z. L. Xie, J. W. Y. Lam, L. Cheng, H. Y. Chen, C. F. Qiu, H. S. Kwok, X. W. Zhan, Y. Q. Liu, D. B. Zhu and B. Z. Tang, Chem. Commun., 2001, 1740-1741.

23 J. W. Chen, B. Xu, X. Y. Ouyang, B. Z. Tang and Y. Cao, J. Phys. Chem. A, 2004, 108, 7522-7526.

24 Y. Ren, J. W. Y. Lam, Y. Q. Dong, B. Z. Tang and K. S. Wong, J. Phys. Chem. B, 2005, 109, 1135-1140.

25 Z. X. Wang, H. X. Shao, J. C. Ye, L. Tang and P. Lu, J. Phys. Chem. B, 2005, 109, 19627-19633.

26 G.-F. Zhang, H. F. Wang, M. P. Aldred, T. Chen, Z.-Q. Chen, X. G. Meng and M. Q. Zhu, Chem. Mater., 2014, 26, 4433-4446.

27 X. J. Xu, J. Huang, J. J. Li, J. W. Yan, J. Q. Qin and Z. Li, Chem. Commun., 2011, 47, 12385-12387.

28 J. Huang, R. L. Tang, T. Zhang, Q. Q. Li, G. Yu, S. Y. Xie, Y. Q. Liu, S. H. Ye, J. G. Qin and Z. Li, Chem. - Eur. J., 2014, 20, 5317-5326.

29 J. Huang, N. Sun, P. Y. Chen, R. L. Tang, Q. Q. Li, D. G. Ma and Z. Li, Chem. Commun., 2014, 50, 2136-2138.

30 J. Huang, N. Sun, J. Yang, R. L. Tang, Q. Q. Li, D. G. Ma and Z. Li, Adv. Funct. Mater., 2014, 24, 7645-7654.

31 J. Yang, N. Sun, J. Huang, Q. Q. Li, Q. Peng, X. Tang, Y. Q. Dong, D. G. Ma and Z. Li, J. Mater. Chem. C, 2015, 3, 2624-2631.

32 S. Odabas, E. Tekin, F. Turksoy and C. Tanyeli, J. Mater. Chem. C, 2013, 1, 7081-7091.

33 B. R. He, Z. F. Chang, Y. B. Jiang, X. F. Xu, P. Lu, H. S. Kwok, J. Zhou, H. Y. Qiu, Z. J. Zhao and B. Z. Tang, Dyes Pigm., 2014, 106, 87-93.

34 W. Z. Yuan, Y. Gong, S. Chen, X. Y. Shen, J. W. Y. Lam, P. Lu, Y. W. Lu, Z. M. Wang, R. R. Hu, N. Xie, H. S. Kwok, Y. M. Zhang, J. Z. Sun and B. Z. Tang, Chem. Mater., 2012, 24, 1518-1528.

35 L. M. Leung, W. Y. Lo, S. K. So, K. M. Lee and W. K. Choi, J. Am. Chem. Soc., 2000, 122, 5640-5641.

36 S. L. Tao, C. S. Lee, S.-T. Lee and X. H. Zhang, Appl. Phys. Lett., 2007, 91, 013507.

37 S. L. Tao, L. Li, J. S. Yu, Y. D. Jiang, Y. C. Zhou, C.-S. Lee, S.-T. Lee, X. H. Zhang and O. Kwon, Chem. Mater., 2009, 21, 1284-1287.

38 C.-L. Liu, C.-J. Zheng, X.-K. Liu, Z. Chen, J.-P. Yang, F. Li, X.-M. Ou and X.-H. Zhang, J. Mater. Chem. C, 2015, 3, 1068-1076.

39 C. W. Tang, S. A. VanSlyke and C. H. Chen, J. Appl. Phys., 1989, 65, 3610-3616.

40 W. Z. Yuan, P. Lu, S. Chen, J. W. Y. Lam, Z. Wang, Y. Liu, H. S. Kwok, Y. Ma and B. Z. Tang, Adv. Mater., 2010, 22, 2159-2163.

41 W. Qin, J. Z. Liu, S. M. Chen, J. W. Y. Lam, M. Arseneault, Z. Y. Yang, Q. L. Zhao, H. S. Kwok and B. Z. Tang, J. Mater. Chem. C, 2014, 2, 3756-3761. 
42 Y. Liu, X. H. Chen, Y. Lv, S. M. Chen, J. W. Y. Lam, F. Mahtab, H. S. Kwok, X. T. Tao and B. Z. Tang, Chem. Eur. J., 2012, 18, 9929-9938.

43 L. Chen, Y. B. Jiang, H. Nie, R. R. Hu, H. S. Kwok, F. Huang, A. J. Qin, Z. J. Zhao and B. Z. Tang, ACS Appl. Mater. Interfaces, 2014, 6, 17215-17225.

44 Z. Y. Yang, Z. G. Chi, T. Yu, X. Q. Zhang, M. N. Chen, B. J. Xu, S. W. Liu, Y. Zhang and J. R. Xu, J. Mater. Chem., 2009, 19, 5541-5546.

45 H. Y. Li, Z. G. Chi, B. J. Xu, X. Q. Zhang, Z. Y. Yang, X. F. Li, S. W. Liu, Y. Zhang and J. R. Xu, J. Mater. Chem., 2010, 20, 6103-6110.
46 J. Y. Kim, T. Yasuda, Y. S. Yang and C. Adachi, Adv. Mater., 2013, 25, 2666-2671.

47 C. Y. K. Chan, J. W. Y. Lam, Z. J. Zhao, S. M. Chen, P. Lu, H. H. Y. Sung, H. S. Kwok, Y. G. Ma, I. D. Williams and B. Z. Tang, J. Mater. Chem. C, 2014, 2, 4320-4327.

48 H. Y. Li, Z. G. Chi, X. Q. Zhang, B. J. Xu, S. W. Liu, Y. Zhang and J. R. Xu, Chem. Commun., 2011, 47, 11273-11275.

49 J.-Y. Hu, Y.-J. Pu, F. Satoh, S. Kawata, H. Katagiri, H. Sasabe and J. Kido, Adv. Funct. Mater., 2014, 24, 2064-2071.

50 H. A. Al-Attar, G. C. Griffiths, T. N. Moore, M. Tavasli, M. A. Fox, M. R. Bryce and A. P. Monkman, Adv. Funct. Mater., 2011, 21, 2376-2382. 